\section{Severe Hyponatremia following Cystoscopy and Urethral Dilation: An Unusual Late Complication}

Sir,

Hyponatremia is an uncommon complication of surgical procedures requiring non-conductive irrigation fluids. The volume and type of irrigation fluids dictate the severity of hyponatremia caused during cystoscopy and transurethral resection of prostate (TURP). The severity of hyponatremia is related to the volume of irrigation fluid retained in body cavities. ${ }^{1}$ The amount of irrigant fluid, required to develop hyponatremia, is comparatively smaller in women. This usually presents within one to two days after surgery. Our patient presented with symptomatic hyponatremia 10 days post-surgery, which is rare.

A 50-year woman with uncontrolled hypertension for 15 years and type 2 diabetes mellitus for three years, was in her usual state of health two weeks back, when she suddenly developed increased urinary frequency with right flank pain. A cystoscopy procedure using hypotonic glycine as the irrigating medium was performed at another centre, to treat urethral stricture and the patient recovered symptomatically. However, 10 days postoperatively, the patient gradually developed drowsiness and was brought to the Emergency Room of our centre. The patient was received in a drowsy condition with a Glasgow coma scale (GCS) of 3/15 and random blood sugar of $190 \mathrm{mg} / \mathrm{dl}$. She did not have fever and had no history of nausea, vomiting, loose stools or urinary symptoms. On examination, the patient had a supple neck, and decreased bilateral plantar reflexes, but normal bilateral pupillary responses to light. She was intubated due to low GCS. An emergent computed tomography (CT) scan of head was performed, which showed no signs of ischemia or hemorrhage. Blood tests and electrolytes were ordered and the patient was started on intravenous (IV) hydration. The complete blood count $(\mathrm{CBC})$ showed normal hemoglobin with a decreased hematocrit $(33.8 \%)$, increased white blood cells $\left(30.1 \times 10^{9} / \mathrm{L}\right)$, increased neutrophils (93.8\%) and increased platelets $\left(458 \times 10^{9} / \mathrm{L}\right)$. There was severe hyponatremia $(104 \mathrm{mEq} / \mathrm{L})$ and hypochloremia ( $69 \mathrm{mEq} / \mathrm{L}$ ). After ruling out stroke and urinary tract infection, the patient was diagnosed with late-onset development of symptomatic hyponatremia, secondary to cystoscopic procedure for urethral strictures. The patient was given hypertonic saline, and she recovered well and was extubated after 48 hours with GCS of 13/15.

Urethral strictures in women are rare and can present as dysuria, incontinence or recurrent urinary tract infection. Initially, minimally invasive procedures, such as dilatation or optic internal urethrotomy are used to treat the condition. These procedures offer faster recovery, minimal scarring, and less risk of infection. The only drawbacks are: risks of hematuria, infection, and problems related to anesthesia. Several surgical procedures, such as cystoscopy, use non-conductive irrigation fluid such as mannitol, sorbitol and glycine to enable vision during the procedure. However, their use has been complicated by hyponatremia, leading to development of TURP syndrome, which can manifest as visual disturbances, confusion and other neurological symptoms. ${ }^{2}$ The amount of irrigant fluid required to develop hyponatremia is smaller in women as compared to men. ${ }^{3}$

Walker et al. described a case of a 7-year child, a known case of cloacal exstrophy, who underwent cystoscopy and percutaneous cystolithotomy for the removal of two bladder stones. A total of $2 \mathrm{~L}$ of freely flowing sterile water was used for bladder irrigation during the procedure. Over a $30-\mathrm{min}$ period, patient became disoriented and ultimately progressed to respiratory arrest. After immediate re-intubation, she began to have seizures and her sodium levels dropped to $120 \mathrm{mmol} / \mathrm{L}$. She was given furosemide and sent for a head CT which showed massive cerebral edema which caused her death. ${ }^{4}$

Demirel et al. reported a case of a 78-year man who was diagnosed with benign prostatic hyperplasia. The patient was scheduled for TURP under general anesthesia. Approximately $42 \mathrm{~L}$ of irrigation fluid was used perioperatively. In the $155^{\text {th }}$ minute of surgery, the patient became cyanotic, bradycardic, and developed hypotension. Blood gas analysis showed sodium levels of $90 \mathrm{mmol} / \mathrm{L}$. The patient was diagnosed with TURP syndrome and was administered diuretics. The patient was discharged without any neurological symptoms and fluid-electrolytes imbalance. ${ }^{5}$

Szolnoki et al. presented a case of a 7-year boy who had lumbosacral myelomeningocele at $\mathrm{L} 5-\mathrm{S} 1$, resulting in neurogenic bowel and bladder dysfunction. Patient developed three episodes of generalised seizures post-cystoscopy. His electrolytes showed a serum sodium level of $119 \mathrm{mmol} / \mathrm{L}$, urine sodium of $38 \mathrm{mmol} / \mathrm{L}$, and serum osmolality of $254 \mathrm{mOsm} / \mathrm{Kg}$. The diagnosis of hyponatremic seizures was made predominantly due to free water intoxication and to a lesser degree inappropriate antidiuretic hormone (ADH) secretion. He was started on $0.9 \%$ sodium chloride and the next day, his serum sodium rose to 129 $\mathrm{mmol} / \mathrm{L}$. The patient was discharged in stablecondition. ${ }^{6}$

Hyponatremia is a known complication of cystoscopy, caused by irrigation fluid. Usually, it manifests in early post-cystoscopy period. Our patient developed hyponatremia 10 days after the procedure, which is really a delayed presentation. Timely recognition and prompt management is required for better outcome.

\section{CONFLICT OF INTEREST:}

The authors declared no conflict of interest.

\section{AUTHORS'CONTRIBUTION:}

MIS: Conceived the idea and wrote firstdraft.

SA: Wrote background and also made addition to discussion.

NA: Reviewed and finalised the letter to editor. 


\section{REFERENCES}

1. Ghanem AN. Postoperative dilution hyponatraemia and the turp syndrome: Critical analytical review of literature on patho-etiology and therapy. EC Emergency Medicine and Critical Care 2019; 3(8):507-14.

2. Rambam Health $C$. The effect of irrigation solution volume on electrolytes and acid-base balance - clinicaltrials.gov. Clinicaltrials.gov. 2015.

3. Istre $\mathrm{O}$, Hahn R. Hyponatremia following transurethral resection or hysteroscopy. Uptodate.com. 2018.

4. Walker MR, Fuhr PG, Koyle MA. Fatal hyponatremia in a 7year-old girl with cloacal exstrophy after cystoscopy for a bladder stone. J Pediatr Urol 2008; 4(3):231-3. doi: 10.1016/j.jpurol.2007.12.004.

5. Demirel I, Ozer AB, Bayar MK, Erhan OL. TURP syndrome and severe hyponatremia under general anaesthesia. BMJ Case Reports 2012; 2012:bcr-2012. DOI: 10.1136/bcr2012-006899.

6. Szolnoki JM, Puskas F, Sweeney DM, Camporesi EM,
Upadhyay J. Hyponatremic seizures after suprapubic catheter placement in 7-year-old child. Paediatr Anaesth 2006; 16(2):192-4. doi: 10.1111/j.1460-9592.2005. 01632.x.

Mir Ibrahim Sajid ${ }^{1}$, Shanila Ahmed ${ }^{2}$ and Noman Ali ${ }^{3}$

${ }^{1}$ The Aga Khan University Hospital, Karachi, Pakistan ${ }^{2}$ Department of Oncology, Section Medical Oncology, The Aga Khan University \& Hospital, Karachi, Pakistan

${ }^{3}$ Department of Emergency Medicine, The Aga Khan University \& Hospital, Karachi, Pakistan

Correspondence to: Dr. Shanila Ahmed, Department of Medical Oncology, The Aga Khan University \& Hospital, Karachi, Pakistan

E-mail: shanilaahmed90@yahoo.com

Received: June 12, 2020; Revised: August 31, 2020;

Accepted: October 01, 2020

DOI: https://doi.org/10.29271/jcpsp.2021.04.493 